\title{
THIRST RESPONSE TO POST-EXERCISE FLUID REPLACEMENT NEEDS AND CONTROLLED DRINKING: COMMENTS FROM REVIEWERS ALONG THE PUBLICATION PROCESS ${ }^{1}$
}

Publication of scientific work in peer-reviewed journals is an imperfect process subject to many criticisms, particularly from authors. At the same time, some scientists complain that many previously rejected papers are eventually published in other journals, often without paying attention to the shortcomings that have been pointed out in the process. As an exercise in transparency, both the authors and the journal, Pensar en Movimiento, invite any interested readers to analyze the present manuscript in the light of all the prior reviews and four rejections. We warn our readers not to make the mistake of judging the reviewers from the perspective of the final, published paper, as it differs considerably from the versions they were asked to evaluate.

Catalina Capitán-Jiménez and Luis Fernando Aragón-Vargas

\section{From the first submission, Appetite, February 2014.}

Ms. Ref. No.: APPETITE-D-13-00763

Title: Thirst sensitivity to post-exercise fluid replacement needs and controlled drinking

Dear Dr. ARAGON-VARGAS,

After careful consideration, I have regretfully had to conclude that this paper is unsuitable for publication and an acceptable presentation of this work is hard to envisage. Any available comments about the submitted manuscript are appended below.

Thank you for giving us this opportunity to consider your work. I am sorry about the disappointing outcome on this occasion. This editorial decision removes access to this paper from the online submission site.

With best regards,

Simon Nigel Thornton, $\mathrm{PhD}$

Co-Executive Editor

Appetite

\footnotetext{
${ }^{1}$ Capitán-Jiménez, C., \& Aragón-Vargas, L. (2016). THIRST RESPONSE TO POST-EXERCISE FLUID REPLACEMENT NEEDS AND CONTROLLED DRINKING. PENSAR EN MOVIMIENTO (Thinking in/about Motion), 14(2), 1-16. doi:http://dx.doi.org/10.15517/pensarmov.v14i2.25853

-1 - 
Reviewer \#1: Abstract, line 19, 43 and 368: The text states that "... we deem thirst to be far from perfect". This is a problematic statement and the data to support this statement is not evident. For decades, authors have said that thirst returns to a near-baseline level, and called this an "early slowing of drinking", which has a purpose(Rolls BJ. Palatability and fluid intake. In: Fluid Replacement and Heat Stress, 1993, pages 161-167. National Academy Press, Washington D.C.). Compare to line 109 and lines 307-309. This purpose is to ensure that thirsty humans do not over-drink, perhaps to the point of illness or water intoxication. It is not clear what the authors expect of thirst. What is "perfect"? How does the finding of a very strong inverse association between thirst and NFB fit here (line 312)? How does a finding of a clear association between hypohydration and thirst (lines 335-336) relate to this statement? This sounds as though thirst is a good index of

NFB, not "far from perfect".

Abstract, line 19 (line 44): The text states that "... [thirst] responds inappropriately". However, thirst decreases after drinking. That is appropriate.

Abstract, line 16: The text states that this thirst scale can detect dehydration equivalent to $2 \%$ BM loss. The text does not state an obvious fact: thirst is not sensed at less than 1-2\% BML. Several points in the text (line 363-364) ignore this obvious point.

Line 64-65: The author's name is misspelled.

Lines 72-84: This text is irrelevant to the purpose and focus of this paper. Please remove this paragraph.

Lines 138-140: Engell's scale should be describe in greater detail.

Lines 153-159 (and Figure 1): What is the role of showering (change in skin temperature) on thermal afferent inputs to thirst? Was the shower water temperature controlled? Was skin temperature measured?

It is obvious that plasma osmolality is an essential, if not the most important input, to thirst. Plasma osmolality was not measured. This should be stated as a limitation of the present study, especially in the multiple regression analysis. What value is a MLR that omits the strongest predictor of thirst? Also, parts of the Discussion section should be modified to acknowledge this omission.

Figures 1 and 2: Looking at the vertical axis, what are the anchors (verbal statements) that link to each number on Engell's scale? Where does thirst appear on this scale (i.e., not thirsty versus somewhat thirsty)? Does \#3 represent the threshold of perceived thirst? This should be considered in the Discussion section, at several points, because it may alter the logic. 
Line 287, 364: How was "robust" determined / defined? Was this a statistical test for robustness?

Line 344: Cite references please.

Reviewer \#2: The manuscript entitled "Thirst sensitivity to post-exercise fluid replacement needs and controlled drinking" examined the accuracy of thirst ratings in different hydration states. The topic of the study is interesting and the questions are important. However, there are several issues that need to be addressed.

General Comments

The manuscript is poorly written and difficult to follow. The introduction is extensive and has a lot of information, beyond the topic of the present study.

The lack of plasma osmolality data in a study examining thirst while blood samples have been collected is hindering the ability to discuss potential physiological mechanisms.

The plasma volume changes data do not look physiologically sound. It is unusual to have none or minor decrease in PV as a response to dehydration and a major plasma volume expansion as a response to partial rehydration with plain water. It is not clear if the authors have standardized for environmental temperature and posture in order to accurately measure hemoglobin and hematocrit. Also the pre exercise baseline blood sample has been taken via direct venous puncture without any indication of use of tourniquet.

The measurements of hematocrit and hemoglobin with an automated systems lacks validity especially when changes in plasma osmolality occur (Watson P, Maughan RJ. Artifacts in Plasma Volume Changes due to Hematology Analyzer-Derived Hematocrit. Med Sci Sports Exerc 46: 52-59, 2013.)

The lack of indication of core temperature and the cold shower prior to the measurement could also blunt some of the thirst responses, since body temperature plays a modulating role both in thirst and anti-diuretic hormone secretion (Takamata A, Mack GW, Stachenfeld NS, Nadel ER. Body temperature modification of osmotically induced vasopressin secretion and thirst in humans. Am J Physiol 269: R874-80, 1995.)

The volunteers of the study were 4 males and 4 females. Since the study is focusing in fluid balance it is important that the menstrual cycle stage is taken into consideration. It is unclear if the authors have tested the females at the same phase of their cycle.

-3 -

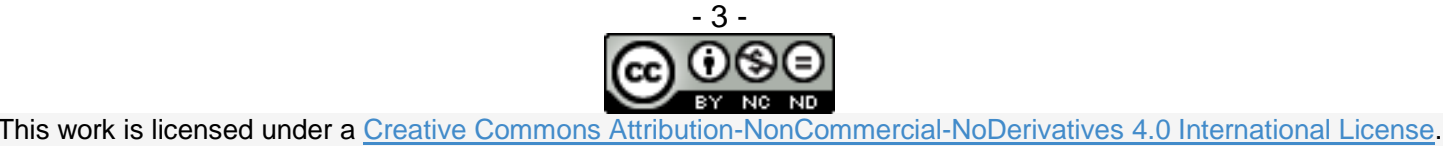


The authors are using for the multiple regression analysis their 8 experimental units (subjects) as 160 experimental observations and they analyze their data based on that, which is a statistical violation.

The line graphs should have error bars on the top of the data points and not on a separate area of the graph as group mean error bar. To avoid clutter you can use for the higher values only positive error bars and for lower values only negative error bars.

The results would be more clearly presented if the authors will use less statistical references.

One of the purposes of the study is to examine the reliability of the thirst scale used. However, no reliability (test, retest under the same conditions) has been reported. It would be better if these data were removed.

\section{From the second submission, European Journal of Applied Physiology, September 2014.}

\section{Ref:}

Ms. No. EJAP-D-14-00545

Thirst sensitivity to post-exercise fluid replacement needs and controlled drinking European Journal of Applied Physiology

\section{Dear Dr. ARAGON-VARGAS,}

Comments have now been received from external reviewers with particular expertise in the area of your manuscript. Although we found the topic of your paper interesting, regrettably the various concerns that we identified preclude your manuscript from attaining a sufficiently high priority to warrant publication in EJAP.

For your guidance, we append the reviewers' comments below, which we hope will prove constructive.

You will see that the first two reviewers differed in opinion, and for that reason we asked for a third view. Unfortunately this was negative.

We greatly appreciate your giving us the opportunity to consider your work, and look forward to having the privilege of reading future manuscripts from you and your colleagues.

Yours sincerely, 
George Havenith, PhD

Editor

European Journal of Applied Physiology

Reviewers' comments:

Reviewer \#1: The authors have conducted this study as part of a larger one investigating the diuretic effect of water; the design is appropriate and the results have been interpreted well, the manuscript written very elegantly with sound philosophical arguments that add an aspect to this paper that make for comforting reading i.e. this topic has received much 'heated' debate from both sides and yet this has a refreshing approach! See below my main comments I have:

1) Abstract - before coming to the Methods, I wondered what the $1.20 \% \mathrm{BM}$ referred to i.e. of body mass or the body mass lost etc. It would serve to highlight this being $1.20 \% \mathrm{BMpre}$ I think to avoid confusion.

2) I find it odd that "voluntary fluid intake" has been determined as a keyword... I can see the authors' logic, however I feel that although the goal of their research programme will shed light on this, given the fluid load was fixed in the current study?

3) I have always been taught that an ICC was a better indicator of reliability than a simple correlation; however, this (as most statistics) is open to interpretation!

4) P-values should be probably be written as $<0.001$ rather than full equation, for readers?

5) For the multiple regression, what does F refer to? Normally beta-coefficients would be described?

6) The authors have done an excellent job of commenting on their limitations in the Discussions whilst still highlighting how their findings progress the field. One remaining omission appears to be the factor of drink temperature - evidence from Sandick (1984), Boulze (1983) and Brunstrom (2002) amongst others have argued/demonstrated that the colder the fluid the more satiating, therefore the fact that you have used $5 \mathrm{C}$ water could inadvertently/artificially have satiated your participants (too quickly/much) compared to say $37 \mathrm{C}$ water?

7) Why do your Table and Fig 1 use SD but Fig 3/4 SEM? Please be consistent.

8) Plasma volume - surely these should be inverted i.e. most be negative as plasma volume is reduced?! 
9) I am unsure how valid your reliability measure is when used within a trial/study such as this. Reliability would normally encompass independent observations i.e. repeated visits as opposed to during the same visit?

Reviewer \#2: This study aimed to determine the sensitivity and reliability of perceived thirst to graded levels of dehydration and post-exercise controlled drinking. The authors concluded that perceived thirst is valid and reliable, but responds inappropriately to water intake after exercise. This is an important area of research with important practical implications; however, this reviewer believes that these conclusions are not supported by the data. In addition, there were major limitations in the study design, which limit the interpretation and applicability of study results.

Abstract, Purpose: The first sentence is background information not a study purpose. Also, the second sentence is too long and difficult to follow. I suggest shortening this sentence or rewriting it as $\sim 2$ short sentences.

Abstract, Line 17: I think "fixed" is a more appropriate word than "pre-determined" here.

Abstract, Line 24-27: This sentence is misleading in that it says the subjects exercised during the $0 \%$ change in body mass trial, but the methods of the paper indicate that subjects rested during this trial.

Abstract, Line 32: It says plasma volume changes were measured, but they were actually calculated.

Abstract, Methods: This section contains no information about how the main outcome variable was measured (i.e., perceived thirst was rated by subjects on a 9-point scale).

Abstract, Results, Line 34: Please include data for thirst.

Abstract, Line 37: Do you mean "significantly" instead of "clearly"?

Abstract, Line 46: You make conclusions about the validity of the scale, but did not say how you measured validity. Besides, isn't this scale already considered to be a valid scale (as indicated in the methods)? Instead, you measured sensitivity to graded dehydration. Abstract, Line 49: How can you conclude that thirst is inappropriate? What is the basis for what is an appropriate response?

Introduction, Page 3, Line 50-52: Please include references to support this statement. Introduction, Page 5, Line 9-38: These two paragraphs are not well written. In particular, the sentence in lines $24-31$ is long and written awkwardly. I suggest rewriting these paragraphs to make them more clear and concise.

Introduction: This section is missing a clear hypothesis.

Methods, Page 6, Line 41-58: Because perceived thirst is influenced by so many factors care should be taken to control and standardize the procedures across all trials. In this study, during the $0 \%$ change in body mass trials (i.e., no dehydration) subjects did not exercise and were not exposed to the hot environment. By contrast they exercised in the heat during the dehydration trials. Furthermore, the subjects' exercise duration varied considerably across dehydration trials. These protocol differences between trials (rest/exercise, exercise duration, environment) could have confounding effects on perceived thirst ratings. These are major limitations of the study

-6 - 
design. Given the study design it is difficult to say whether thirst changed because of dehydration per se or because of these other potential confounding factors.

Methods, Page 7, Line 6: Was exercise intensity consistent across trials for each subject?

Methods, Page 8, Line 14: The lack of plasma osmolality measurement is a significant

limitation. Thirst is more sensitive to plasma osmolality changes than plasma volume changes at these relatively low levels of dehydration tested.

Methods, Page 8, line 24-31: This sentence is confusing.

Methods, Page 8, line 34-39: The method of measuring the reliability of the thirst scale is questionable. Please provide a supporting reference or explanation for how pre- to post-shower measurements represent similar conditions to measure reliability. Also, what is the day-to-day reliability of the measure? Why not compare the reliability of measures taken at baseline of each trial to determine the method reliability? This would seem to be more useful than pre- to postshower reliability.

Results, Page 9, line 45: How did thirst compare from baseline to post-rest in the 0\% BM trial? Did subjects shower during this trial as well?

Results, Page 10, line 21: Net fluid balance is written out here but abbreviated elsewhere. The manuscript is very inconsistent throughout in the use of abbreviations. For example, see PV and plasma volume written out on page 12 and see TP and thirst perception written out on page 11 . Results, Page 10, line 48: Were these other factors: subjects, condition, and time, significant predictors of thirst? What is the correlation between perceived thirst and exercise duration? Is the duration of exercise (across trials) a significant predictor of thirst?

Results, Figure 4: Please indicate on the figure when drinking took place. At 0 min the level of dehydration was minimal - only 0 to $1.5 \%$ body mass deficit. These are low levels of dehydration and it has been shown previously that thirst is often not perceived at these low levels.

Results, Table 1: There is a 0.5 a.u. difference in baseline thirst between $1 \% \mathrm{BM}$ and all the other trials. This seems like a significant difference at baseline considering that it is only a 9-pt scale. This represents a $20 \%$ discrepancy. Can you explain?

Discussion: Overall there should be less discussion on the debate around thirst and more talk of the scientific support/data behind the arguments.

Discussion: Here and throughout the paper the writing is too conversational in places. For example, page 15, line 33 ("thirst is far from perfect"), page 14, line 36 ("works well for athletes").

Discussion, Page 15, line 19: It is a major overstatement to say this 1 study confirms anything, especially given the methodological limitations outlined above. This paper does not provide strong support in favor of thirst being "inappropriate".

Discussion, Page 15, line 21: This statement is written as if two separate tests were run to determine reliability and how "robust" the thirst scale is. The same test (pre- to post-shower thirst) can't be used to measure two separate things?

Discussion, Page 15, line 34: What is meant by "responds inappropriately"? Do you mean insensitive? Please consider more careful word selection. Your manuscript title includes sensitivity, but you make no conclusions regarding sensitivity. I suggest discussing whether

-7 - 
perceived thirst is sensitive to changes in hydration status, rather than focusing on the debate of what is "appropriate".

\section{Reviewer \#3: GENERAL}

The purposes of this study were to determine if a relationship exists between thirst and dehydration ("does thirst distinguish among several levels of dehydration?") and to determine the thirst response to a standard ingested water volume (satiation). The authors also state the desire to assess the reliability of Engell's (1987) thirst scale. The 'true' novelty of any part of this study is highly nuanced. There are also several conceptual, methodological, and analytical limitations. Specific questions and comments follow for consideration.

\section{MINOR}

Introduction - The authors set-up the justification for their paper by citing confusion over whether or not drinking "to thirst" is acceptable during exercise. However, their study design has nothing to do with testing the efficacy of drinking during exercise. A better rationale should be written to support the study design chosen. For example, begin with work done by Denton (e.g., satiation of thirst - PNAS, 1999 - 2014), which is incredibly not recognized (!), and build from there.

Methods - please elaborate on when urine samples were obtained given the timing of blood draws and the need for controlled posture.

Results - why not evaluate the relationship (correlation) between thirst and dehydration level using regression (prediction)?

The authors acknowledge that this study was part of a larger experiment, but that does not itself make limitations acceptable.

It is inconceivable that a paper on this topic would not reference E. Rolls or D. Denton. See also great texts on thirst by Denton and Chapter 15 (Thirst, Adolph and Wills) in Adolph, Physiology of Man in the Desert (1947).

\section{MAJOR}

General - what does this study teach that was not already known about thirst and dehydration? The answer is not clear to me.

Concept - a basic understanding of tonicity and volume influences on thirst tells us that thirst is driven principally by Posm (not measured) and secondarily by blood volume. The latter does not reliably stimulate thirst until approximately $10 \%$ of volume is lost from the vasculature (e.g., 
Cheuvront and Kenefick, Comp Phys 2014 - review of basic physiology). Why then would such small anticipated changes in PV be suspected to relate to thirst in the first place?

Methods and Discussion - The authors lament over why they chose plain water and the volume given. Their justifications are curious. What does palatability have to do with anything when the volume ingested is 'prescribed?' Further, ACSM fluid intake guidelines (2007) do not suggest $100 \%$ replacement of sweat losses during exercise - only prevention of excessive dehydration ( $>2 \%$ body mass). Fluid volume ingestion 'after' exercise depends on the timing of the next exercise bout (e.g., Shirreffs, 2004), among other things. The authors do not seem to fully understand the recommendations or the issues.

Statistics - some justification for sample size and anticipated magnitude of effects should be given.

Results - was reliability between post-exercise and post-shower thirst (not ideal, incidentally) calculated using ICC? Since baseline thirst was measured on 4 occasions, perhaps a coefficient of variation for resting thirst ratings would help establish the score required to exceed, say, 2xSD of $\mathrm{CV}(\mathrm{P}<0.05)$. The observed level of dehydration observed to produce such a rating could then be objectively evaluated against the threshold to establish the utility of the arbitray units (just an example - see Cheuvront et al. AJCN 2010). This would be more insightful information about Engell's scale.

Results - the increase in PV with dehydration of 1-2\% body mass is a troubling methodological shortcoming.

Results - a rule of thumb for multiple regression is to have 10 subjects per predictor variable of interest. That would require 50 subjects. Perhaps just consider the strength of relationships to thirst using individual variables, particularly as so many you have selected are co-related (covary).

\section{From the third submission, Journal of Sports Sciences, December 2015.}

(9 days after submission, apparently a decision from the editor in charge without sending out to reviewers)

Dear Dr. Aragón-Vargas:

I write you in regards to manuscript \# RJSP-2015-1308 entitled "Thirst response to post-exercise fluid replacement needs and controlled drinking" which you submitted to the Journal of Sports Sciences. 
In view of the criticism that the work contained within the manuscript does not provide a significant amount of new information to progress the area of study, it will not be possible to consider further your manuscript for publication in the Journal of Sports Sciences.

Thank you for considering the Journal of Sports Sciences for the publication of your research. I hope the outcome of this specific submission will not discourage you from the submission of future manuscripts.

Sincerely,

Prof. Gareth Davison

Section Editor, Journal of Sports Sciences

gw.davison@ulster.ac.uk

\section{From the fourth submission, Journal of Sport and Health Science. June 2016}

\section{7-Jun-2016}

\section{Dear Ms. Capitán-Jiménez,}

Your manuscript entitled "Thirst response to post-exercise fluid replacement needs and controlled drinking" was reviewed by three experts in the field and the corresponding expert associate editor for JSHS. Unfortunately, the review panel did not provide sufficient support for your manuscript to be further considered for publication in JSHS. Although the reviewers appreciated the work that went into this particular study, they had concerns regarding the gender differences in thirst perception, the originality of the results, and the lack of well-defined scientific goals and hypotheses.

Please note that JSHS receives many more manuscripts than we can publish. Therefore, only manuscripts that receive full support from the review panel and are deemed to have the potential to make a significant impact in the field are forwarded for publication.

Let me take this opportunity to thank you for having chosen JSHS as the potential outlet of your research. We appreciate your support and hope that you may consider JSHS for some of your future work.

Sincerely

Walter Herzog (co-editor in chief, JSHS) 
Reviewer(s)' Comments to Author:

\section{Reviewer: 1}

Comments to the Author

General comments

The goal of this study was to determine if perceived thirst could distinguished between euhydration and hypohydration of 1, 2 and 3\% during a recovery period of $3 \mathrm{~h}$ following exercise when a fixed-volume of water ingestion (1.20\% body mass) was consumed over a 30 min period. 8 physically active students ( 4 men and women) were recruited. The authors concluded correctly that thirst perception is a valid index of hydration in the absence of drinking but it responds inappropriately to water intake. The article is well-written, concise and within the scope of JSHS. The present Reviewer has several concerns about this article that precludes its acceptance in the current form. One major concern of this Reviewer is that 4 women were included in the study and that care was apparently not taken to ensure that they were in the same phase of their menstrual cycle when they underwent the study. It is well-known that menstrual cycle has an impact on perceived thirst, and if this factor was not controlled for, then findings may have been confounded by this factor.

\section{Major comments}

- It is indicated that the reliability of the perceived thirst scale was assessed. However, the timespace between measurements was only $30 \mathrm{~min}$. It is unclear whether this finding is useful in this context. Reliability would have been best assessed if all subjects would have undergone the same experiment several days apart. As such, I am recommending removing this variable from this study as, additionally, it detracts readers from the main goal of the study.

- 4 women were included in the study and care was apparently not taken to ensure that they were in the same phase of their menstrual cycle when they underwent the study. It is well-known that menstrual cycle has an impact on perceived thirst, and if this factor was not controlled for, then findings may have been confounded by this factor. Did menstrual cycle was controlled for in the current study? If yes, please indicate. I recommend looking at this article: Exerc Sport Sci Rev. 2008 Jul; 36(3): 152-159.

- Why were subjects in the 2 and $3 \%$ hypohydration trials not hypohydrated by at least that amount prior to rehydration? See Table 1. Please explain.

- Figure 4 is confusing, and it does not add significantly to the article. Hence, I suggest removing it, and instead to replace it by a correlation between perceived thirst and net fluid balance, which is the best predictor of perceived thirst. 
- Page 9, Lines 11-16: I do not understand why the power analysis was computed using urine volume. It should have been measured using thirst sensation.

Minor comments

- Page 3, Lines 25-30: please add a reference

- Page 3, Lines 30-32: the goal of drinking to thirst during exercise is exactly not to maintain euhydration, such to stay away from the adverse effect of hyponatremia. What do you mean by observation?

- Page 3, Lines 35-40: this is not totally correct. Please see those studies and adjust accordingly.

Dugas JP, Oosthuizen U, Tucker R, Noakes TD. Rates of fluid ingestion alter pacing but not thermoregulatory responses during prolonged exercise in hot and humid conditions with appropriate convective cooling. Eur J Appl Physiol. 2009 Jan;105(1):69-80.

Lee MJ, Hammond KM, Vasdev A, Poole KL, Impey SG, Close GL, Morton JP. Self-selecting fluid intake while maintaining high carbohydrate availability does not impair half-marathon performance. Int J Sports Med. 2014 Dec;35(14):1216-22.

Dion T, Savoie FA, Asselin A, Gariepy C, Goulet ED. Half-marathon running performance is not improved by a rate of fluid intake above that dictated by thirst sensation in trained distance runners. Eur J Appl Physiol. 2013 Dec;113(12):3011-20.

- Page 3, Lines 40-42: The truth is that the impact of drinking to thirst on performance has received few attention, but all studies completed on this topic so far point to the same direction and have demonstrated that it is optimal for performance, which is not the case for the $2 \%$ body mass loss strategy. It appears important to discriminate between the impact of drinking to thirst during exercise vs. post exercise, where the goal of fluid replacement may be different.

- Page 5, Lines 25: what was the mean number of days between each visit?

- Page 6, Lines 10-13: please describe the breakfast in detail.

- Page 6, Lines 25: replace he or she by participants. Please correct everywhere.

- Page 6, Lines 30: why thirst was not measured during exercise?

- Page 7, Lines 19: why did you decide to replace $1.20 \%$

- Page 8, Lines 47: please be consistent and use the abbreviations throughout the text. 


\section{Reviewer: 2}

Comments to the Author

Introduction

Could perceived thirst be called thirst through the manuscript?

The definition of thirst, as the main focus of this study, should be presented earlier in the introduction.

Reference for paragraph 2, first sentence?

Paragraph 3 is not clear- what are the causes for thirst?

It is not clear in the introduction why the post-exercise period is of interest and studied?

Parts of the introduction could be moved to or/and discussed in the discussion, such as in page 4 paragraph 3 (causes for why fluid intake affects the thirst, independent of hydration status).

Aim of the study- what was the overall aim of the study?

\section{Methods}

What was the definition of healthy and of physically active?

Is the larger study published? How does the intervention with diuretics affect the participants this study?

What is non-consecutive days- why not show what days with a figure (dehydration conditions)?

What was the reference method for dehydration?

How did you weigh participants (+scale name, reference)?

What was the calculation for the \%BM?

You talk about a chamber in the methods- what was this chamber for?

What was the equation used to calculate the water volume to be ingested by each individual?

What was the calculation used for the net fluid balance?

What was the analyses made from the $5 \mathrm{ml}$ blood sample?

The standardized breakfast, is it a common breakfast used in sports studies, reference?

The thirst scale- describe more about the procedure of asking about thirst. Is the question for how thirstu they are now or for the last hours or weeks? Did the participants fill in the scale or who did?

I suggest to add a figure on the procedure for the different exercises, dehydration levels and thirst measurements.

Page 7, paragraph 3- missing text end of the sentence "... according to..."

It is not clear why hemoglobin was analyzed? Did you measure serum osmolality?

\section{Discussion}


Authors start to discuss methods, I suggest to start to discuss the main results instead.

Authors should discuss the possible reason why thirst is "diminished" after ingestion of water and not associated with the fluid loss (e.g. receptors in mouth/throat sensing water).

Page 11, last paragraph starts in the middle of a sentence.

Page 13, last paragrapg, first sentence, something is missing.

\section{Reviewer: 3}

Comments to the Author

Investigations about dehydration and rehydration could indicate beneficial points for athletes and deserves the attempt. About this manuscript, the method was performed well but the findings and method and discussion of the subject renders more work and novelty. Thirst rating during dehydration and rehydration has been reported before. Many of the previous studies demonstrate the fact that thirst satiation, and alteration of level of thirst-related-hormones are not proportional to the subjects' fluid balance, but are influenced by fluid volume and temperature, fluid flavor, the act of swallowing which is probably originating from oropharyngeal receptors and other similar parameters. Parameters such as fluid osmolarity or plasma volume restoration are less effective, when short term post-rehydration events are assessed (1-13). I am hopeful as you continue this work, with more novel data and a better writing and English editing, later you accomplish publishing a highly valued paper in this field.

\section{References}

1. Deaux E. Thirst satiation and the temperature of ingested water. Science. 1973;181(4105):1166-7.

2. Hosseinlou A, Khamnei S, Zamanlu M. The effect of water temperature and voluntary drinking on the post rehydration sweating. Int J Clin Exp Med. 2013;6(8):683-7.

3. Hosseinlou A, Khamnei S, Zamanlu M. Voluntary drinking versus imposed drinking in the methodology of investigations about the drinking-induced thermoregulatory sweating. International journal of clinical and experimental medicine. 2014;7(10):3757.

4. Khamnei S, Hosseinlou A, Ibrahimi H. The effect of volume of consumed water on drinking-induced sweating and plasma levels of arginine vasopressin, epinephrine and norepinephrine. International Journal of Endocrinology and Metabolism. 2004;2004(1, Winter):19-28.

5. Khamnei S, Hosseinlou A, Zamanlu M. Water temperature, voluntary drinking and fluid balance in dehydrated taekwondo athletes. Journal of sports science \& medicine. 2011;10(4):718-24.

6. Millard-Stafford M, Wendland DM, O'Dea NK, Norman TL. Thirst and hydration status in everyday life. Nutrition reviews. 2012;70(suppl 2):S147-S51. 
7. Obika L, Okpere S, Ozoene J, Amabebe E. The role of oropharnygeal receptors in thirst perception after dehydration and rehydration. Nigerian Journal of Physiological Sciences. 2015;29(1):37-42.

8. Riebe D, Maresh CM, Armstrong LE, Kenefick RW, Castellani JW, Echegaray ME, et al. Effects of oral and intravenous rehydration on ratings of perceived exertion and thirst. Medicine and science in sports and exercise. 1997;29(1):117-24.

9. SALATA RA, VERBALIS JG, ROBINSON AG. Cold Water Stimulation of Oropharyngeal Receptors in Man Inhibits Release of Vasopressin*. The Journal of Clinical Endocrinology \& Metabolism. 1987;65(3):561-7.

10. Shirreffs SM, Merson SJ, Fraser SM, Archer DT. The effects of fluid restriction on hydration status and subjective feelings in man. British Journal of Nutrition. 2004;91(06):951-8. 11. Takamata A, Mack GW, Gillen CM, Nadel ER. Sodium appetite, thirst, and body fluid regulation in humans during rehydration without sodium replacement. The American journal of physiology. 1994;266(5 Pt 2):R1493-502.

12. Verbalis J. Inhibitory controls of drinking: satiation of thirst. Thirst: Springer; 1991. p. 313-34.

13. Wilk B, Bar-Or O. Effect of drink flavor and $\mathrm{NaCl}$ on voluntary drinking and hydration in boys exercising in the heat. Journal of Applied Physiology. 1996;80(4):1112-7.

\section{Editor(s)' Comments to Author:}

Associate Editor

Comments to the Author:

Thank you for submitting manuscript number JSHS-2016-0222 to the Journal of Sport and Health Science titled: Thirst response to post-exercise fluid replacement needs and controlled drinking. The authors present a study of an interesting topical area regarding if perceived thirst could distinguish between euhydration and hypohydration of 1,2 and 3\% during a recovery period of $3 \mathrm{~h}$ following exercise. The manuscript was reviewed by three individuals with expertise in this area. Unfortunately, the manuscript did not receive scores high enough for publication. The reviewers expressed several reasons for a low score;

1. Because thirst is a main focus of this study, the introduction should contain a proper definition for thirst.

2. The authors must clarify in the introduction why the post-exercise period is of interest and studied.

3. Also in the introduction the overall aim of the study is not clearly present. The authors must clarify their purpose.

4. Methodological issues; use of women without taking care to ensure that they were in the same phase of their menstrual cycle during the study.

5. The authors indicated that the reliability of the perceived thirst scale was assessed, but this assessment in the context of the purpose of this study adds little. 
6. Figure 4 needs clarification and should be removed, and be replace by a correlation between perceived thirst and net fluid balance.

7. Other methodological issues have been identified that warrant the authors' consideration and explanation (see reviewer comments).

For detailed explanation of each reviewers' comments and questions, please see their remarks. 